\title{
Coulisses
}

Revue de théâtre

43 | Automne 2011

Reviviscences de Faust

\section{Faust Fragments. Penser les théâtres de Pessoa avec le comparatisme}

Julia Peslier

\section{(2) OpenEdition}

1 Journals

Édition électronique

URL : https://journals.openedition.org/coulisses/225

DOI : 10.4000/coulisses.225

ISSN : 2546-9460

Éditeur

Presses universitaires de Franche-Comté

Édition imprimée

Date de publication : 31 décembre 2011

Pagination : 49-60

ISBN : 978-2-84867-404-9

ISSN : $1150-594 X$

Référence électronique

Julia Peslier, "Faust Fragments. Penser les théâtres de Pessoa avec le comparatisme », Coulisses [En ligne], 43 | Automne 2011, mis en ligne le 30 novembre 2016, consulté le 29 décembre 2022. URL

http://journals.openedition.org/coulisses/225 ; DOI : https://doi.org/10.4000/coulisses.225 


\title{
Faust Fragments. Penser les théâtres de Pessoa avec le comparatisme
}

\author{
Julia Peslier
}

\section{Pessoa face à la tentation comparatiste}

1 Auteur de l'inachevé Fausto, tragedia subjectiva, Pessoa nomme un héros comme réincarné du Faust I et II de Goethe dans la langue portugaise. Il le voue à un genre nouveau : celui de la "tragédie subjective ", qui n'est ni tout à fait un monologue de théâtre, ni un théâtre statique tel Le Marin. Comme un crâne de pensées sur le point de se faire et de se défaire en Faust, où les voix de Christ, de Bouddha, de Shakespeare et de Goethe viennent côtoyer celle du Mystère du monde, de Maria, de Lucifer, du Vieux et encore des Disciples, la fragmentaire tragédie du sujet accroît singulièrement la teneur de solitude : celle du héros, celle du lecteur et du spectateur, celle encore que la critique prête au poète portugais en personne. Or Pessoa, pour nous autres, c'est aussi l'orchestration hétéronymique, sonore dans le monde de la critique et révélée comme géniale. Se rêvant littérature à soi seul, dans un texte fameux, un Projet de préface à un livre futur ${ }^{1}$, Pessoa, face à la pénurie de littérature portugaise qu'il diagnostique, est-il à prendre au sérieux ? Inventant un remède fondé sur la pluralité auctoriale, il partage l'œuvre en œuvres, selon un mouvement de genèse singulier conté dans ses lettres à Côrtes Rodrigues et à Adolfo Casais Monteiro ${ }^{2}$, si fréquemment citées. En ces terres, chaque œuvre prend le nom d'un poète, dans l'épreuve de leur simultanéité stimulante: Pessoa l'orthonyme, lui-même, parait sur la scène poétique en réaction au Maître Caiero, qui a également motivé la puissance du verbe de Campos, quand Reis s'illustrera plus tard. L'hétéronymie est aujourd'hui un phénomène bien connu, digne d'un manuel scolaire. Fausto, pourtant, résiste encore, tel un contrepoint qui mettrait en crise le discours sur l'hétéronymie du côté de la critique.

2 C'est là que commence notre réflexion sur les théatres de Pessoa ${ }^{3}$ et leur face à face avec le comparatisme, là également que son Fausto trouve son actualité. CEuvre inachevée, exigeante et magnifique, elle est un défi pour l'éditeur, le metteur en scène et le philologue qui souhaitent en donner une actualisation. Lire, monter et jouer 
Fausto, c'est également situer cet ensemble de fragments dramatiques dans l'œuvre plus vaste de Pessoa, ainsi que dans la bibliothèque faustienne et son héritage. C'est à coup sûr prendre des décisions herméneutiques dans la réception pessoenne, faire de la théorie littéraire, se soucier de philologie et de dramaturgie tout ensemble. Comment appréhender l'œuvre de Pessoa, si frappée du sceau de l'hétéronymie ? Y a t-il là autant d'îlots à considérer sur le mode "À chaque poète son œuvre et son commentaire inhérent $»^{4}$ ? Ou bien s'agit-il de le lire dans sa totalité, comme la geste de quelque auteur-secrétaire, poète inventeur de poètes? Le panorama qui se dessine est celui d'une critique kaléidoscopique, qui multiplie les visions de l'œuvre (où la pulsion scopique est fragmentée et rassemblée en un même entrelacs, pour former un seul bel objet critique, dit " pessoen ») et qui dans le même temps s'enclôt comme une critique monographique des œuvres hétéronymiques (qui, de Caeiro, de Soares, de Reis, de Campos ou de Pessoa orthonyme, parmi d'autres possibles ${ }^{5}$, déploie des esthétiques, des styles, des poèmes de facture et d'influences diverses). Avec parfois des propositions plus retorses au plan biographique et auctorial - le rêve de faire de Pessoa et de ses autres une invention de Borges ${ }^{6}$, celui de faire de Tabucchi, son commentateur zélé, un énième hétéronyme ${ }^{7}$, celui, aussi, de l'écrivain Saramago, d'écrire la biographie de l'hétéronyme Ricardo Reis (O Ano da morte de Ricardo Reis, 1984), celui peut-être de Patrick Quillier, de faire tendre le traducteur vers un autre hétéronyme, celui encore, énoncé par Michel Deguy, d'être soi-même, lecteur, à la fois un hétéronyme possible de Pessoa et l'auteur d'un de ses poèmes ${ }^{8}$.

Les critiques inventent un comparatisme interne à l'œuvre en quelque sorte, d'où la littérature mondiale disparaît comme sous le débord et la densité hétéronymiques, quand la littérature comparée qui goûte le loisir de rapprocher Pessoa des autres monuments de la modernité ${ }^{9}$ pourrait ramasser l'hétéronymie à une invention d'auteur, si géniale soit-elle, mais qui serait toujours à lire comme œuvre de Pessoa et donc ne créditerait pas sérieusement un comparatisme entre Caeiro et Virgile, Walt Whitman ou Cesário Verde comme auteurs d'égale facture et en ce sens affaiblirait la puissance du crédit accordé à l'invention pessoenne ${ }^{10}$. CEuvre de portée plus confidentielle, plus rare dans le commentaire comme sur la scène, Fausto offre un laboratoire passionnant pour mener cet état des lieux de la critique pessoenne et de sa tentation comparatiste. Trois mouvements nous permettront d'initier cette réflexion sur les fragments dramatiques faustiens. Le premier sera une brève synthèse des inventions de la critique pour nommer la dramaturgie poétique pessoenne, ce vaste théâtre en œuvres, et la tentation de lire l'hétéronymie en Fausto. Le deuxième montrera comment, à travers le travail original de Teresa Rita Lopes, grande spécialiste de l'œuvre et elle-même dramaturge, s'est configuré un univers éditorial très singulier autour des textes inédits de Pessoa. Le troisième se focalisera sur le Fausto, pour en présenter des montages de Teresa Sobral Cunha et de Patrick Quillier, aussi originaux qu'incompréhensibles peut-être si l'on ne tenait pas compte de la spécificité de cette œuvre et de sa réception.

\section{Du Théâtre Pessoa à Faust (tragédie subjective) : le report hétéronymique}

4 Il s'agit d'esquisser les termes, les arborescences et les rameaux d'une généalogie critique sur la figure de Pessoa, oscillant entre unité et pluralité auctoriales, afin de 
mettre au jour un report hétéronymique sur le héros faustien, un réinvestissement voire une projection du quatuor Reis, Caiero, Campos et Pessoa dans la figure solitaire du penseur Faust. La réception française est initiée par Armand Guibert, qui intitule sa préface au Gardeur de troupeaux (Gallimard, «nrf Poésie», 1987) "Visages avec masques ", réactivant l'étymon latin, persona, "masque » de théâtre, qui produisit le nom commun pessoa portugais («personne» au sens de quelqu'un). Teresa Rita Lopes aura une influence majeure par son travail, sur laquelle nous reviendrons, jouant notamment sur la dramaticité de l'hétéronymie comme mode d'entrée et de pratique théâtrale dans l'œuvre - elle crée des dialogues sur le mode ludique pour construire une herméneutique. Dans sa lignée, José Augusto Seabra forge le néologisme et concept du poemodrama qui produit le poetodrama pour définir l'hétéronymie ${ }^{11}$ (il s'agit d'un essai tiré de sa thèse dirigée par Roland Barthes et soutenue en 1971); ailleurs il évoquera l'« hétérotexte pessoen" (O Heterotexto pessoano, Dinalivro, 1985). António Tabucchi, dans le domaine italien, mais immédiatement actif sur les réceptions portugaises et françaises notamment, relaie des mythographies pessoennes: Un baule pieno di gente. Scritti su Fernando Pessoa, Sogni di sogni, I Dialoghi mancati - Il Signor Pirandello è desiderato al telefono. Il fictionne des fragments de vie pessoens, il opère l'exercice critique en prenant littéralement acte des dispositifs par lesquels Pessoa fait entrer en fiction ses poètes et ses hétéronymes; en un sens Antonio Tabucchi cartographie l'œuvre en se fondant sur sa double activité de critique et d'écrivain.

De son côté, biographe de Pessoa et commentateur de longue date, Robert Bréchon se concentre sur le poète comme sujet humain et manie le paradoxe pour compenser l'involution fictive des textes pessoens: ses biographies reflètent ce mouvement, prenant pour titres: Étrange étranger: une biographie de Pessoa (Bourgois, 1996); L'Innombrable. Un tombeau pour Fernando Pessoa (Bourgois, 2001), Pessoa le poète intranquille (Aden, 2002), Fernando Pessoa, le voyageur immobile (Aden, 2002). Il intitule l'anthologie de poèmes par Pessoa par la citation Je ne suis personne activant le jeu de mot bilingue entre pessoa, une personne, et le terme français personne (qui vaut, sur le mode ulysséen, l'outis grec ou le nemo latin); il hétéronymise Pessoa et Kafka par le poème «Franz Pessoa et Fernando Kafka » (L'Innombrable) et, enfin, construit l'expression $d u$ «Pessoa réel » quand il déploie en même temps une lecture très rimbaldienne, proche du Je est un autre, de l'œuvre. Par sa thèse de doctorat en philosophie soutenue en 1997 sous la direction de Philippe Lacoue Labarthe, relayée dans son séminaire au Collège de philosophie, Judith Balso défend la thèse d'un Pessoa le Passeur métaphysique, posant la question de la philosophie et de la poésie, montrant que sa poésie est "pensée de la poésie comme pensée » et que chaque hétéronyme se définit esthétiquement, ontologiquement, par rapport au Maître Caiero. Il y aura bien d'autres approches encore à rappeler, notamment celle passionnante de Leila PerroneMoisés (Fernando Pessoa Aquém do Eu, Além do Outro, Marins Fontes, 1982) ou de José Gil (Pessoa ou a Metafísica das Sensações, 1987).

De cette synthèse, un principe ressort, c'est la puissance du dispositif hétéronymique sur le commentaire. Or cette dynamique va nous seulement investir l'espace de la publication des œuvres, et notamment des fragments dramatiques, mais elle infléchira également la lecture et les montages des fragments faustiens. Longtemps de nature hétéronymique, l'entrée dans l'œuvre de Pessoa trouve un étonnement, un émerveillement aussi, à découvrir dans le Fausto le resserrement à un sujet, qui se définit en des qualificatifs proches du Desdichado de Nerval-«Eu sou o Aparte, o 
Excluído, o Negro ! [Je suis le Solitaire, l'exclu, le Ténébreux !] » ${ }^{12}$, assumant la marge de la pensée, son mouvement aussi lancinant que celui des marées anxieuses de la pensée (à l'ouverture du Fausto), sa brisure perpétuelle sur les rivages qu'elle aborde. Au passage, la critique découvre le péril de pluralisation à l'extrême de l'auteur, voire de pulvérisation de ses écrits en une infinité d'éditions de florilèges, de fragments, de collections. Il y a alors la tentation de lire en Fausto un autre de ces théâtres de Pessoa dont l'inachèvement intrinsèque, le chaos manuscrit et la désorganisation dramatique apparente portent la marque de la pensée plurielle, composite, fragmentée - en somme hétéronymique, dans le même temps que le héros faustien, dans ses magnifiques apories et métaphores de la pensée en acte, sera identifié à Pessoa en personne, voire, ultime boutade, à personne.

7 Eduardo Lourenço décrit cet « échec apparent, ou même objectif » comme « une ruine à nu, sans envers ", " son Waterloo ", créant le mot composé « Pessoa-Faust » et allant jusqu'à considérer que la tragédie subjective aura « fait de Fernando Pessoa le Faust de lui-même $~^{13}$. Judith Balso lit en Fausto, (tragedia subjectiva) le revers de l'hétéronymie poétique, la poursuite aporétique de sa passion pour la métaphysique (d'où son inachèvement constitutif), quand l'hétéronymie en signifierait une résolution positive, poétique ${ }^{14}$. Patrick Quillier (responsable du volume en Pléiade des Cuvres poétiques de Pessoa), avance le terme extrêmement productif d'acroamatique dans le colloque de Cerisy: Fernando Pessoa: unité, diversité, obliquité, pour penser la pluralité vocale du texte, son hétérogénéité intrinsèque de pensées. Acroamatique, "cette tentative d'audiocritique doit bel et bien pouvoir être nommée méthode acroamatique dans la mesure où elle privilégie l'oreille comme instance centrale de l'expérience littéraire, chez l'écrivain comme chez son lecteur ${ }^{15}$, vient également de la grande pratique de la traduction, dont Patrick Quillier connaît les théories et la pratique. Manuel Gusmão défend en Fausto un poème impossible ${ }^{16}$, dont il étudie les antagonismes, les dualismes, les apories. L'inachèvement de la pièce est reconnu comme essentiel, le mode aporétique du questionnement faustien tenant lieu d'impossibilité à conclure, de cycle indécidable de la pensée. Pascal Dethurens analyse le rapport du sujet de l'énonciation faustienne comme centrifuge et mise en spectacle de soi-même, là où Pessoa n'est plus paraît alors Faust, Pessoa (n') étant personne :

Il y a, à l'origine de la création pessoenne, et surtout dans son Faust, la béance du moi [...]. Celui qui écrit et celui qui pense, chez Pessoa, n'est personne. [...] Il demeure néanmoins ceci de plus important dans l'invention théâtrale pessoenne, qu'il y a mise en spectacle de soi-même, c'est-à-dire mise en théâtre de l'homme. [...]

Tout y est, tout le sens de la «tragédie subjective», se concentre en cette superbe strophe, qui apparaît comme la déclinaison du nom propre de l'écrivain: Pessoa, c'est personne ${ }^{17}$.

8 C'est ainsi que la question centrale, esthétique, de l'unité et de la pluralité du poète s'est localisée dans la lecture du Faust.

\section{Un art du montage pour du théâtre en fragments}

9 Le mouvement de fragmentation et de montage est plus général, il vaut pour l'ensemble de l'œuvre. À ceci s'ajoute l'orphelinage des œuvres inachevées : faisant face au défaut de l'auteur, à la lisibilité délicate des manuscrits, la philologie et le travail éditorial viennent en compensation. Et à force d'asserter la synonymie bilingue entre Pessoa et personne, on a fini par réduire l'écrivain, comme entité auctoriale, à néant ; on 
a même pu alors commencer à s'en passer, s'autorisant de son injonction même, pour pouvoir le publier sans lui, afin de lui redonner, en un retour paradoxal, une œuvre, qui n'est plus tout à fait la sienne, sans être totalement d'un autre. Le geste inaugural est donné par Teresa Rita Lopes, qui soutient sa thèse "Fernando Pessoa et le drame symboliste » en 1975, sous la direction de René Étiemble, à la Sorbonne. Elle redouble ce geste herméneutique par la proposition étonnante du Théâtre de l'Être génériquement défini comme des "textes rassemblés, traduits et mis en situation». On y trouve des citations des divers hétéronymes pessoens, de Faust, de Soares, ainsi que tout un appareil de didascalies, qui peut à l'occasion disposer une scène d'écriture (Pessoa) ou ménager des rencontres et des dialogues improbables entre les hétéronymes et les œuvres. Improbable, bien sûr, si l'on se fie au dispositif de lecture configuré par Pessoa - Faust n'y dialogue pas avec Caeiro par exemple, ils appartiennent à des univers de fictions, à des diégèses poétiques distinctes. Si Pessoa, par la Discussion en famille et les Notes en souvenir de mon Maitre ${ }^{18}$, rendait pensable la rencontre entre les hétéronymes Caiero, Campos, Reis et Pessoa notamment, rien n'indiquait que celle entre Faust et Caiero ou Campos et Reis puisse avoir lieu, y compris poétiquement. Cela revient d'ailleurs à faire du Faust un auteur à part entière, au même titre que les autres, ou, au rebours, à considérer les poètes hétéronymes comme fictions de bibliothèque au même titre que Faust - c'est-à-dire sans leur accorder le supplément d'autorité qui les fait poètes.

Dans son mémoire de thèse, elle note ainsi à propos du théâtre inédit qui recouvre une dizaine de pièces dramatiques :

Tels qu'ils étaient réunis, ces textes paraissaient des pièces à peine ébauchées : rien ne les reliait, ils semblaient les pièces éparses d'un puzzle incomplet. On pourrait même douter que ce puzzle ait jamais constitué une unité que ce soit au niveau des réalisations de Pessoa ou même à celui de ses projets. Dans l'absence d'une véritable histoire, d'un motif, ce puzzle est bien plus difficile à assembler. C'était en quelque sorte un tableau abstrait que l'on devait reconstituer. Car ce qui intéressait Pessoa n'était pas l'affabulation. Une grande partie de ses pièces a été conçue comme les hétéronymes: il s'agissait d'un "monologue prolongé ", jamais clos, poursuivi parfois à deux ou trois voix. Une fois créés, les personnages se manifestaient de façon intermittente. Comme Faust, ils ont tous écrits à leur façon le journal intime que Pessoa n'a jamais tenu ${ }^{19}$.

11 Puzzle, labyrinthe, valse, jeu de patience, voilà autant de termes étonnants qu'on pourra lire dans les appareils critiques de ces théâtres inédits pour qualifier le geste d'édition, le parcours de lecture ou la forme de l'œuvre. Ils sont les signes manifestes que la philologie invente de nouvelles pratiques pour publier les œuvres inachevées de Pessoa, prenant appui sur des spectacles, des créations artistiques, des soirées-lectures pour trouver des formes adéquates ou, du moins, poétiques et dramatiques ${ }^{20}$ qui leur donne une intensité chaque fois actualisée.

Figure de proue des études pessoennes, Teresa Rita Lopes ouvre ainsi une voie possible pour de nouvelles éditions, qui ne séparent pas l'activité théâtrale, sa pensée propre de jeux avec les masques, ses dispositifs scéniques, ses trouvailles en matière de dramatis personae et l'édition scientifique, a priori philologique puisqu'elle concerne un travail sur manuscrits et tapuscrits, ainsi que ses modalités de présentation et de destination aux lecteurs, voire ses prédilections théoriques (conception aristotélicienne du drame, modernité poétique et fragmentation, intertextualité, etc.). Elle posera un nouveau jalon avec une autre édition bilingue, chez Corti, dans la collection Ibérique : Le Privilège 
des chemins, montage de fragments, tirés de quatre pièces dramatiques (Dialogue dans le jardin du Palais, Mort du Prince, Salomé et Calvaire ou Christ).

\section{Faust-fragments}

13 Du paysage éditorial du Faust, on présentera trois éditions issues des réceptions portugaise et française. La première édition intégrale du dossier conservé à la Bibliothèque Nationale du Portugal est celle de Teresa Sobral Cunha (Presença, 1988; Bourgois, 1990), dont tous s'accordent à souligner la grande rigueur, la pertinence et l'intelligence du texte dramatique. Elle se place dans une paradoxale reconstitution de ce que la tragédie aurait pu être et reprend l'architecture en cinq actes d'une tragédie classique, avec prologue et épilogue, s'achevant - au delà de l'inachèvement même du corpus - par la mort du personnage éponyme. Sans être véritablement génétique, elle joint dans l'édition portugaise les plans d'œuvres, des notes préparatoires, quelques fac-similés précieux pour se faire une idée du texte. Elle fait signe vers du théâtre à lire plutôt - ou autant - qu'à jouer. Elle figure son geste comme tissage de Pénélope, une œuvre de couture face à des fragments désassemblés. C'est cette édition qui sert ici de texte-témoin, comme dans une pratique expérimentale.

Or un autre Fausto, leitura em 20 quadros de Pessoa, également agencé par Teresa Sobral Cunha, apparaît en 1994 au Portugal, chez l'éditeur indépendant de poésie, d'essais et de littérature Relógio d'Água, avec un sous-titre programmatique et une préface essentielle pour saisir la nature du montage dramatique. C'est un composite des pièces faustiennes pessoennes - le conte en prose A Hora do Diabo est fondu dans la tragédie en vers Fausto - ainsi que des didascalies de Teresa Rita Lopes qui font appel à l'imaginaire faustien (le Faust, eine deutsche Volkssage de Murnau notamment en un geste de comparatisme externe) et où les actes et scènes annoncés par Pessoa disparaissent au

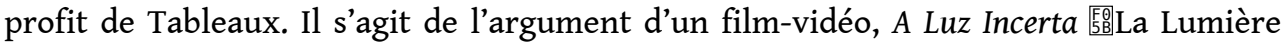
incertaine㹂, réalisé par Margarida Gil en 1994. Elle part de la circulation possible entre les deux œuvres qui ont en commun la figure féminine, Maria, et l'apparition fugitive, à un bal, de deux hommes costumés en Faust et en Diable (opérant un comparatisme interne à l'œuvre pessoenne). De là, elle fonde une circularité et une généalogie entre les deux œuvres, selon un principe ordonnateur de réécriture et un jeu de puzzle où l'acteur jouant Faust tiendrait en puissance tous les rôles masculins. Pourtant, d'après le Dramatis Personae, l'acteur incarnant Faust et le Diable tient beaucoup de Pessoa et l'actrice jouant Maria prend les traits d'Ophélia Queiroz, l'éternelle fiancée du poète portugais : le geste d'ouverture de Teresa Sobral Cunha sur la bibliothèque faustienne est alors strictement replié sur l'iconographie pessoenne. Elle fait du poète Pessoa, le père, le fils (porté par Maria et qui deviendra à son tour poète génial), l'amant, le diable, le personnage, l'acteur l'incarnant et l'auteur, en une complexe superposition.

Chandeigne publie un autre état du Faust pessoen, œuvré et traduit par Patrick Quillier en vue d'une création, à Vienne, en 1994, pour la troupe, Le Théâtre du Paradoxe, qu'il dirigeait. Partant d'une "une analogie certaine $»^{21}$, entre Faust et le dispositif hétéronymique et sensible à l'argument de Lourenço ( faire de Pessoa le Faust de luimême »), Patrick Quillier s'autorise de la première édition de Teresa Sobral Cunha pour présenter ce nouveau travail dramaturgique des fragments faustiens. Son hypothèse est qu'il est difficile d'attribuer à une seule voix le dialogue de Faust, fait de voix plurielles et de fragments disparates, d'où cette démultiplication de Faust en rôles distincts. Plus 
original est le mouvement comparatiste qu'il met à l'œuvre en appliquant du Bachelard et son analyse de la "vieille partition en Animus et Anima» au personnage de Faust, qu'il reconfigure en quatre entités à l'instar de l'hétéronymie restreinte des quatre poètes Reis, Campos, Caiero et Pessoa :

Il nous a semblé légitime d'incarner les quatre pôles à l'œuvre dans la subjectivité faustienne en quatre "dramatis personae»: Faust «Animus », en quelque sorte Faust orthonyme; Faust-Anima, l'«âme» extériorisée qui fait de la femme intérieure une utopie ou un phantasme ; Faust-Lucifer, tout rayonnant comme un ectoplasme de lumière noire; et Faust-Vénus, qui est un double luciférien de l'Anima, une Anima perverse, une " âme damnée », et même " condamnée »"2.

Le travail sur le dramatis personae est fondé sur le quatuor d'acteurs: le quatuor hétéronymique (masculin) et le quatuor faustien (pour moitié féminin), auxquels viennent s'ajouter les autres voix, les personnages et le chœur. L'architecture se fonde sur quatre parties, découpées respectivement en six, vingt-deux, onze et treize séquences, un épilogue. La pièce débute par la Taverne, avec la question redoublée de la nomination et de l'identification du héros : «-Docteur Faust ? / (FAUST) : Oui. / - Le Docteur Faust / (FAUST) : Oui, le Docteur Faust ; qu'y a-t-il d'étonnant à l'être ? $»^{23}$. La Séquence 6 , Partie 1 , est explicative de l'hétéronymie: phénomène extrinsèque à Fausto, mais que Patrick Quillier remet ici en situation d'ouverture, scindant les fragments liés à la Taverne, puisque la chanson du Bon Buveur, écho goethéen de la Chanson de la Puce et des Chansons à boire (In Taberna dans les Carmina Burana) inaugurera la Partie 2.

17 Véritable montage entre l'hétéronymie et la tragédie subjective, théâtre dans lequel Pessoa (l'acteur) évolue à son tour, parlant d'égal à égal avec ses créatures, la pièce conçue par Patrick Quillier va jusqu'à en orchestrer le dialogue impossible, le redoublement par répons et écho autour de questionnements pessoens - par exemple celui sur l'être, dont Hamlet n'est jamais bien loin dans le Fausto. C'est là un détail intéressant de comparatisme interne à l'œuvre dans la dramaturgie : il met en scène comme un bégaiement hétéronymique, une variation sur le verbe être (recommencé ensuite sur celui d'Existence) afin d'introduire ce mystérieux personnage tiré du dramatis personae faustien :

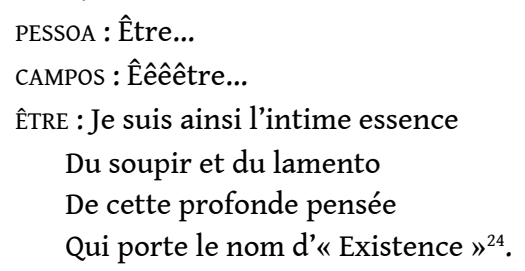

18 On assiste là à la mise en théâtre de ce comparatisme interne, propre à l'œuvre pessoenne, où l'hétéronymie est partout mise sur écoute et où Campos incarne les avant-gardes modernistes, l'ironie, la vocifération, la verve de l'onomatopée. Et voici un exemple d'usage dramatique qui relèverait d'un comparatisme externe: Patrick Quillier fait dire par Goethe et Pessoa (en chorus) une même réplique - «Et le monde stupéfait sent / comme est beau ce que je lui ai donné. $»^{25}$

D'autres déplacements conséquents ont lieu quand on se soucie d'herméneutique faustienne : Lucifer est en Faust, il est un Faust-Lucifer et non une entité séparée - c'est là une vieille question du duel Faust/Méphisto, qu'on ne résout pas si facilement en plaçant le diable à l'intérieur de l'homme comme un double, la complexité en étant tout autre (une complicité de langage, une maïeutique par le questionnement critique du 
diable). Pessoa apparaît comme l'auteur de Faust (Séquence 11, Partie 3) : il en dit l'une des didascalies, quand Faust s'évanouit et tombe à même le sol dans un clair de lune spectral. À la fin, Pessoa devient Faust, il advient en sa parole au moment précis où il dira le texte de Faust, texte fameux parce que celui où le héros énonce son projet d'œuvre-qui est un projet d'écriture. La question qu'il porte-«Écrire mais qu'écrirais-je ? $»^{26}$ est bien celle de Faust au-delà même de l'œuvre pessoenne. C'est celle d'un Faust Phoenix qui revient sur la scène littéraire après Goethe, renait de ses cendres et renoue avec la mélancolie et la vacuité du savoir, prononçant la difficulté à produire de l'inédit dans la pensée. Elle est pourtant complétée par la manière de réponse suivante: I I know not what tomorrow will bring. $"{ }^{27}$, qui sont les dernières paroles attribuées à Pessoa, en un retour sempiternel à l'autobiographie. Le tout est suivi de la didascalie: "Mort, enterrement et résurrection de Pessoa ${ }^{28}$. Symboliquement, Patrick Quillier fait de Pessoa-et non de Faust-son Phoenix. L'acteur jouant Pessoa rejoue, en deux vers, et le drame de Faust et le dernier éclair de conscience de l'écrivain, pour à son tour, comme acteur, mourir, être enterré et entrer en résurrection - sur scène.

Il y a loin, de la dramaturgie d'un Fausto comme chambre parcellaire des échos hétéronymiques à la confidence intime de la tragédie du sujet Pessoa, de la radicalité d'une lecture faustienne, qui lirait la pièce comme un îlot, un texte inouï, défait et nonhétéronymique, astre chu et constellation de pensée brisées, à un comparatisme des Faust de la bibliothèque mondiale qui en manifesterait l'extrême originalité, dans son œuvre de fragmentation de la figure et de l'érudition goethéenne. Le texte porte pourtant toutes ces acceptions. Il serait aussi difficile d'en finir avec un tel héros chez Pessoa : s'il y avait place, dans ses plans où il projetait trois Faust, pour le Faust-Phoenix face au Faust-legend (goethéen) et quand certains commentateurs y ajoutent le PessoaFaust, il y aura désormais également à compter avec le Faust-fragment, expression à la manière des magnifiques Kafka Fragmente de György Kurtag qui formule cette consistance et cette résistance à l'unicité d'une figure. Tel est le propre de Faust, dans la continuation de Goethe, figure dont la facture est d'être elle-même un livre, un condensé et un devenir fragment de la bibliothèque au sens défini par Barthes dans $L a$ Préparation du roman. En Pessoa, il est éclats de pensée, esquilles de savoir, travail de la pensée à l'œuvre, indéfiniment. Figure de la réincarnation littéraire depuis Goethe, ce qu'avait pointé Valéry dans sa "Préface au lecteur de bonne foi et de mauvaise volonté " ("Mon Faust »), Faust devient ainsi ces poèmes dramatiques qui résistent à une lecture qui ne serait pas, au commencement, acte, décision philologique, mise en action de l'herméneutique. Chez Pessoa, c'est jusque dans la philologie, qui en agence les fragments définitifs, qu'il en vient à trouver son actualité, sa manière d'être, sur le théâtre de la pensée. Il est ainsi, toujours mouvant et présent, « notre Faust ».

\section{NOTES}

1. Fernando Pessoa, "Projet de préface à un livre futur ", in Le Chemin du serpent, Christian Bourgois Éditeur, « Titres », 1991, p. 239. 
2. Lettres à Adolfo Casais Monteiro, ibid., p. 191-220.

3. Ces théâtres seront d'abord entendus au sens large comme théâtre hétéronymique propre à sa poésie, ce qu'il a appelé le Drame en personnes (Drama em gente), puis ils seront considérés au sens restreint des fragments dramatiques, ces pièces inachevées conservées à la Bibliothèque Nationale du Portugal.

4. Je me réfère ici à un entretien de Borges, qui définit les écrivains anglophones comme insulaires, tels des îles dont l'œuvre s'originerait dans la singularité, par opposition aux écrivains français, du moins certains d'eux, qui créeraient de l'impulsion d'un mouvement (esthétique, artistique, littéraire).

5. Ils seraient quelques soixante-dix et poussières, d'importances diverses. Voir le passionnant projet en ligne Pessoa. O Labirinto. Portail MultiPessoa : http://multipessoa.net.

6. Ce rêve est assez récurrent, on le retrouve, par exemple récemment, sous la plume de Jérôme de Gramont, "Sur Fernando Pessoa, "De combien de vies sommes-nous capables ?" ", S.E.R./ Études, 2011/4, tome 414, p. 512.

7. Emmanuel Bouju, «Portrait d'Antonio Tabucchi en hétéronyme posthume de Fernando Pessoa : fiction, rêve fantasmagorie ", Revue de Littérature Comparée, 2003/2, n³06.

8. Michel Deguy, «Projet perpétuel », Rue Descartes, 3004/3, n 45-46, p. 58-74 : « Nous nous y retrouvons. "Je" pourrais être un de ses poèmes/poètes; ou lui un des miens. Comme s'il m'avait prévenu, lu et pastiché à l'avance. Je suis lu dans ses livres. Je suis un de ses hétéronymes. Nous sommes tous des hétéros dans la mesure où à la fois, nous n'existons pas assez et existons possiblement, multiplement, virtuellement. "Je" lui attribue parfois un poème. "

9. Des lectures comparatistes ont ainsi pu être envisagées avec Kafka, Musil, Canetti, Valéry, Cendrars, Joyce, Apollinaire, Michaux - pour ne citer que quelques noms avec lesquels on l'a rapproché.

10. Geste comparatiste qu'il cherche lui-même à créditer : voir, par exemple, le texte « Com quem se pode comparar Caeiro ? Com pouquíssimos poetas. " [" À qui peut-on comparer Caiero ? À très peu de poètes. »] (Je traduis), in Fernando Pessoa, Páginas Íntimas e de Auto-Interpretação, textos estabelecidos e prefaciados por Georg Rudolf Lind e Jacinto do Prado Coelho, Lisboa, Ática, 1996, p. 343.

11. José Augusto Seabra, Fernando Pessoa ou le poétodrame, Corti, «Ibériques », 1988, p. 17 : « Nous sommes là, simultanément, devant un drame en poèmes-ce que nous appellerons le poémodrame - et devant un drame en poètes un poétodrame. Mais ce qu'il faut essentiellement remarquer, c'est le fait que le poétodrame n'est que l'éclatement dans une pluralité de sujets poétiques du drame en poésie, c'est-à-dire, de la structure dramatique de l'œuvre des hétéronymes (le poémodrame).» [José Augusto Seabra, Fernando Pessoa ou o poetodrama, São Paulo, Perspectiva, 1974]

12. Fernando Pessoa, Fausto, tragedia subjectiva (Fragmentos), Edição de Teresa Sobral Cunha, Lisboa, Editorial Presença, 1988, p. 16 ; Faust, tragédie subjective, traduit par Pierre Léglise-Costa et André Velter, Paris, Christian Bourgois Éditeur, 1990. Soulignons ici l'emploi du verbe ser, de l'Essence, par rapport à estar qui aurait signifié un état transitoire : Faust est le solitaire, par essence.

13. Eduardo Lourenço, « Pessoa ou le Vertige ontologique », in ibid., respectivement p. 7, 8, 21, 23.

14. Judith Balso, Pessoa ou le passeur métaphysique, Seuil, «L'Ordre Philosophique », 2006, p. 221.

15. Patrick Quillier, «La Dramaturgie paradoxale de Faust: "tragédie du sujet" et tragédie de l'oreille », in Fernando Pessoa : Unité, diversité, obliquité, Christian Bourgois Éditeur, p. 434, ainsi que " Hétéronymie, traduction et acroamatique », in ibid., p. 391-406.

16. Manuel Gusmão, O Poema impossível : o Fausto de Pessoa, Caminho, 1986.

17. Pascal Dethurens, «Le Faust de Pessoa : poétique et dramaturgie », in ibid., p. 314-315. 
18. Fernando Pessoa, ibid., p. 241-278, pour les deux textes ainsi que Notes en souvenirs de mon Maître Caiero, présentés par Teresa Rita Lopes, traduits par Dominique Lecomte, Fischbacher, « Minuit rouge », 1996.

19. Teresa Rita Lopes, Pessoa et le drame symboliste, Fondation Calouste Gulbenkian/Centre Culturel Portugais, 1985, p. 121-122.

20. Pour les différents gestes et les figures de philologues propres à l'édition pessoenne, je me permets de renvoyer à mon travail « Déraison et invention de la philologie : ou comment donner à lire Pessoa aujourd'hui », LHT, n5, publié le 24 novembre 2008 [En ligne],

URL : http://www.fabula.org/lht/5/64-peslier.

21. Patrick Quillier, «Le Faust de Pessoa ", in Faust, La Mort du Prince, traduits et présentés par Patrick Quillier, Éditions Chandeigne, 1994, p. 8.

22. Ibid., p. 9-10.

23. Ibid., p. 13.

24. Ibid., p. 44.

25. Ibid., p. 35.

26. Ibid., p. 128.

27. Ibid.

28. Ibid.

\section{INDEX}

Palavras-chave : literatura comparada, heteronomia, fragmento

Palabras claves : literatura comparada, heteronomia, fragmento oeuvrecitee Faust

Keywords : comparatism, heteronym, fragment, work in progress

Mots-clés : comparatisme, hétéronymie, fragment, work in progress

\section{AUTEURS}

\section{JULIA PESLIER}

Université de Franche-Comté. Centre Jacques Petit (ELLIADD)

Maître de conférences en littérature comparée 